- Outlines how dentistry can be practised in an evidence-based manner.

- Closes the gap between dental research and day-to-day dental practice.

\title{
Questions and answers in evidence-based patient care
}

\author{
C. Sathorn ${ }^{1}$ and P. Parashos ${ }^{2}$
}

Evidence-based healthcare has become the mainstream of current healthcare practices, yet there seem to be many misunderstandings concerning this concept. This paper reviews several aspects of the concept of evidence-based healthcare in a simple question-and-answer format. The areas considered include: the significance of the evidence-based concept in clinical practice, the method of conducting a detailed electronic search of the literature, and the interpretation and application of research evidence to patients and immediate clinical applications.

\section{INTRODUCTION}

The concept of evidence-based healthcare has assumed major importance in providing optimal treatment modalities for patients. ${ }^{1}$ From one perspective, 'evidence-based' is practically a buzzword in healthcare circles because it is spoken of, quoted, and used as a basis for clinical arguments. However, there appear to be many misunderstandings concerning the concept of evidence-based healthcare, and this may result in the term being abused. The purpose of this article is to dispel any confusion concerning the concept of evidence-based healthcare in a simple question-and-answer format covering concepts, methods, and interpretation with the ultimate goal of improved patient care.

\footnotetext{
1*PhD Scholar; ${ }^{2}$ Senior Lecturer Endodontic Unit; School of Dental Science, The University of Melbourne, Endodontic Unit, 720 Swanston Street, Carlton, Victoria, 3010, Australia

*Correspondence to: Dr Chankhrit Sathorn

Email:sathornc@unimelb.edu.au
}

\section{WHAT IS EVIDENCED-BASED HEALTHCARE?}

It is an integration of best available research evidence with clinical expertise and patient values. ${ }^{1}$ When these three elements are combined, clinicians and patients form a diagnostic and therapeutic alliance which optimises clinical outcomes and quality of life.

\section{WHY EVIDENCE-BASED?}

Life is full of choices. What are we having for dinner tonight? Where are we going shopping tomorrow? These choices can be made simply by deciding what one wants. However, when we are faced with more complex situations, what we want is probably not enough. What brand of composite do we choose over another? Which treatment regimen should be offered to our patients and on what basis? We need solid foundations on which our decisionmaking process can rely. Evidence-based healthcare concepts suggest that these foundations are: 1) research evidence, 2) clinical expertise (eg operator capability and satisfaction) and 3) patient values (eg patient satisfaction, cost).

Clinicians are continually overwhelmed with an unmanageable amount of healthcare information from a variety of sources. In dentistry, there are over 500 journals publishing over 43,000 research articles a year. ${ }^{2}$ How do we cope with this amount of information and yet still be able to provide the current best available treatment to our patients? Evidence-based dentistry (EBD) can help, at least in part.

\section{HOW DOES THIS CONCEPT HELP CLINICIANS AND THEIR PATIENTS?}

It helps to improve the decision-making process making it more objective, consistent, and up-to-date. This ultimately improves the quality of treatment the clinician can provide and the level of healthcare for the patient.

\section{HOW DO WE ACTUALLY PRACTISE EBD?}

Essentially it consists of five steps:

a) Converting the need for information (about prevention, diagnosis, prognosis, therapy, causation, etc) into an answerable question

b) Tracking down the best evidence with which to answer that question

c) Critically appraising that evidence for its validity (closeness to the truth), impact (size of the effect), 


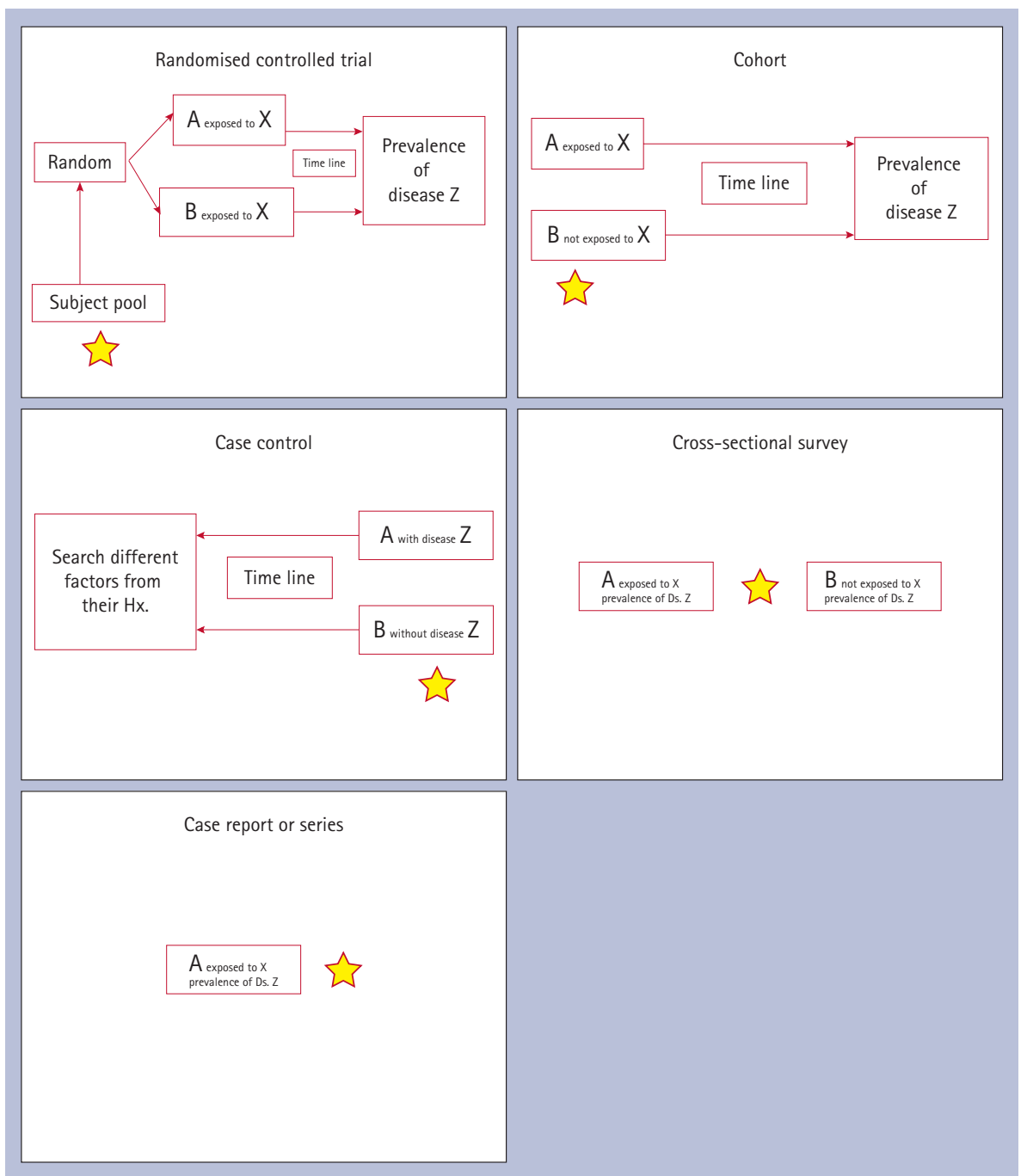

Fig. 1 Different types of clinical studies. The star represents a starting point, $X$ is an affecting factor, $\mathrm{Z}$ is a disease, $\mathrm{A}$ is an experimental group, $\mathrm{B}$ is a control group

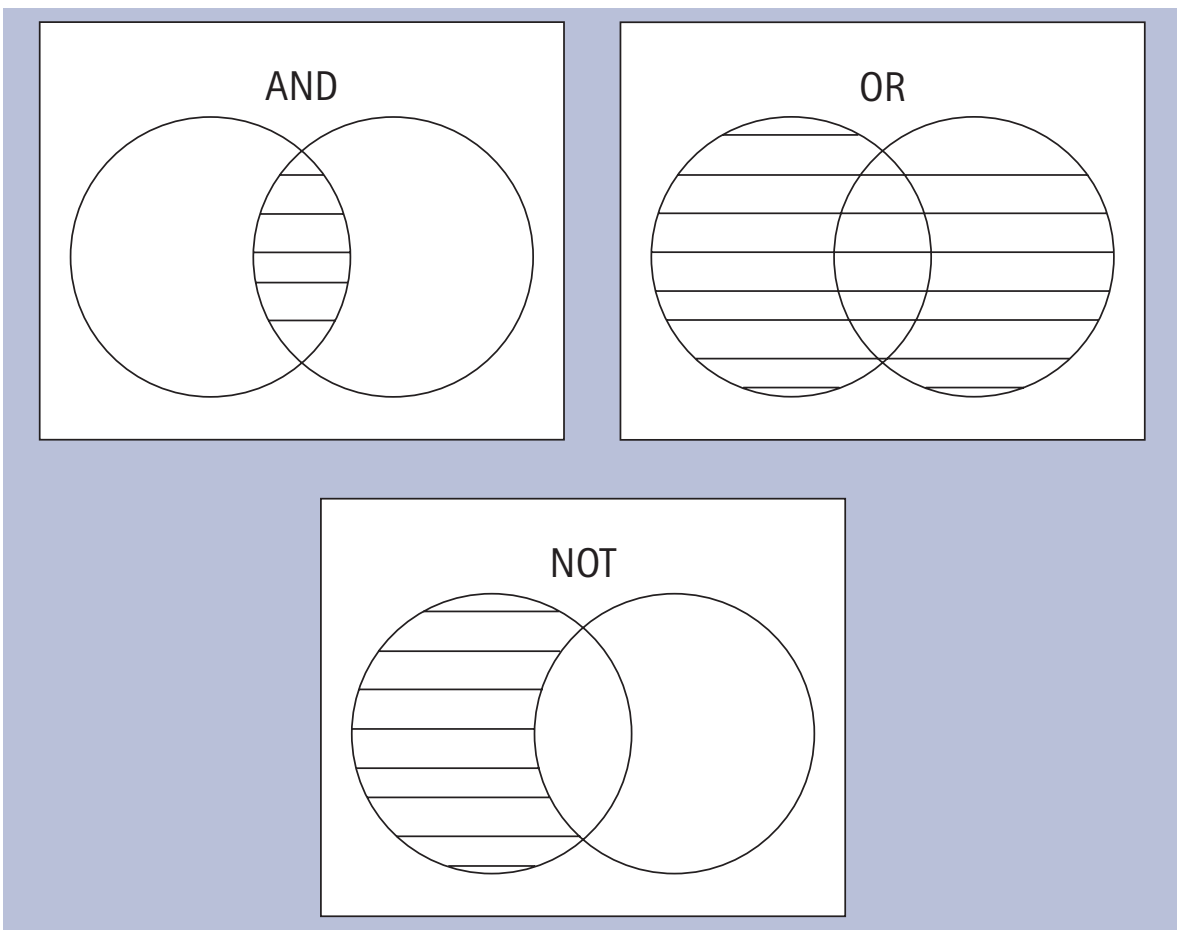

Fig. 2 Pictorial explanation of how Boolean operators work. The circles represent words or phrases and the shading indicates the extent of overlap of these as determined by the three Boolean operators and applicability (usefulness in our clinical practice)

d) Integrating the critical appraisal with our clinical expertise and with our patient's unique biology, values and circumstances

e) Evaluating our effectiveness and efficiency in executing steps a)-d) and seeking ways to improve these for the next time.

\section{WHAT IS THE QUESTION?}

Asking the right question is the first step in getting the right answer. The evidence-based concept suggests that the question should be formulated in the form of a PICO (Problem, Intervention, Comparison, and Outcome) question, ${ }^{3}$ which makes the question clearly defined and more likely to be answerable.

An example of such a question is: In patients undergoing endodontic treatment for apical periodontitis (teeth with an infected root canal system), does single-visit endodontic treatment without a calcium hydroxide dressing, compared to multiple-visit endodontic treatment with a calcium hydroxide dressing for one week or more, result in a lower healing ('success') rate (as measured by clinical and radiographic interpretation) $?^{4}$ In this question the Problem is apical periodontitis, the Intervention is endodontic treatment; the Comparison is single and multiple-visit endodontic treatment, and the Outcome is healing rate of two treatment regimens.

\section{WHAT IS EVIDENCE?}

Simplistically, evidence is everything. Articles appearing in peer reviewed journals are evidence. Systematic reviews and randomised controlled clinical trials are evidence. Expert opinion or even our experiences with individual patients are evidence. The key is their order or their credibility as is explained below.

In order to determine what kind of evidence is more reliable consider the following comparisons.

\subsection{Systematic study vs. clinical impression and/or experience}

Bias cannot be controlled effectively in clinical impression and/or experience.

Operator bias: one clinician's treatment outcomes are better than others. It has been shown that dentists are less likely to prescribe amalgam refilling of their own fillings compared to fillings 
done by others. ${ }^{5}$ Radiographically, endodontic lesions tend to heal more promptly if the operators read their own films. ${ }^{6}$ This bias can be reasonably eliminated in systematic studies.

Population bias: patients are not randomly selected from the population and are not randomly distributed in the dentist's interventions, and, so, generalisation or extrapolation to other clinician's patients are unlikely to be valid. ${ }^{7}$ This bias can be reduced or eliminated in some study designs (eg randomised control trials).

In non-controlled environments (eg private practices) correlation and causation cannot be distinguished. For example, haemorrhagic fever prevalence is very high in the rainy season. Does rain cause haemorrhagic fever? A reasonable person would say 'unlikely'. Because fever prevalence and quantity of rain correlate, that does not mean they are cause and effect. Factors affecting treatment outcomes are much more complex than this example. Clinicians can be deceived easily by correlation. For example, after using Product $\mathrm{X}$ in ten patients, the clinician finds that a week later all endodontic symptoms have disappeared. This is a desirable result, but what does it really mean? Does Product $\mathrm{X}$ resolve endodontic symptoms? Should we always use Product $\mathrm{X}$ ? The truth is that it means nothing, or virtually nothing. The main reason is because there is no control group. Hence, it is not known whether without Product $\mathrm{X}$ all symptoms would have resolved anyway? Again, systematic studies in a controlled environment can reduce or eliminate bias and/or confounders.

\subsection{Scientific logic}

In dentistry, where clinical trials are expensive, time-consuming and not popular, good quality evidence is lacking. Direct evidence is generally unavailable. For example, sodium hypochlorite $(\mathrm{NaOCl})$ has been used in endodontics for decades, yet we do not have direct evidence indicating the benefits of $\mathrm{NaOCl}$ in the improvement of treatment outcomes. Why do we still use $\mathrm{NaOCl}$ when direct evidence is lacking? The answer is scientific logic. Although direct evidence is lacking, and the association of treatment success with the use of $\mathrm{NaOCl}$ has never been established, this does not preclude us from logical thinking.
Here is an example of a simple logical thought process: Apical periodontitis is caused by bacteria. ${ }^{8-10}$ Removal of the cause should cure the disease. This notion is also substantiated by a study showing that bacteria-negative canals have a better chance of healing (disease cured). ${ }^{11} \mathrm{NaOCl}$ can eliminate bacteria. ${ }^{12}$ Therefore, because the cause is removed, the disease should then be cured. This thought process is termed scientific logic. This is also considered evidence. In dentistry, scientific logic is used extensively because direct evidence is scarce, albeit much more reliable and more clinically relevant.

\subsection{Clinical vs. laboratory studies}

Of the articles published in the Journal of Endodontics during 1989-1990, $21.3 \%$ were laboratory leakage studies. ${ }^{13}$ This figure indicates that leakage studies comprise a major part of endodontic research, and this is likely to be true even now. The results of those studies can be so contradictory that very few conclusions can be drawn, if at all. For example, one laboratory study concluded that root canals can be completely contaminated after bacterial challenge in 19 days. ${ }^{14}$ But what does this actually mean clinically? Does it mean retreatment of every root canal after gutta-percha is exposed to saliva for 19 days? A clinical study, on the contrary, has shown otherwise, ${ }^{15}$ specifically that the loss of the coronal restoration had little impact on the healing rate or endodontic success. Even though the sample size was much too small to draw a definitive conclusion, the data in this paper suggested (and only suggested) that the problem of coronal leakage may not be of such a great clinical importance as implicated

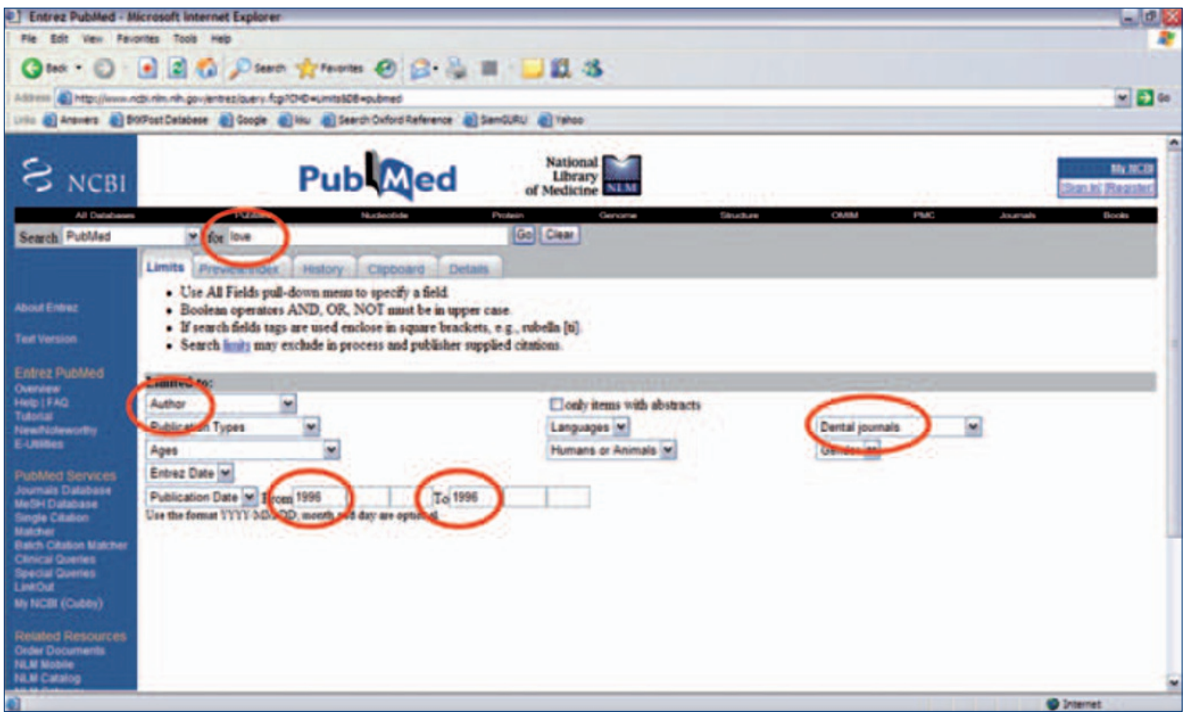

Fig. 3 Locating an article already known to exist. See text for explanation

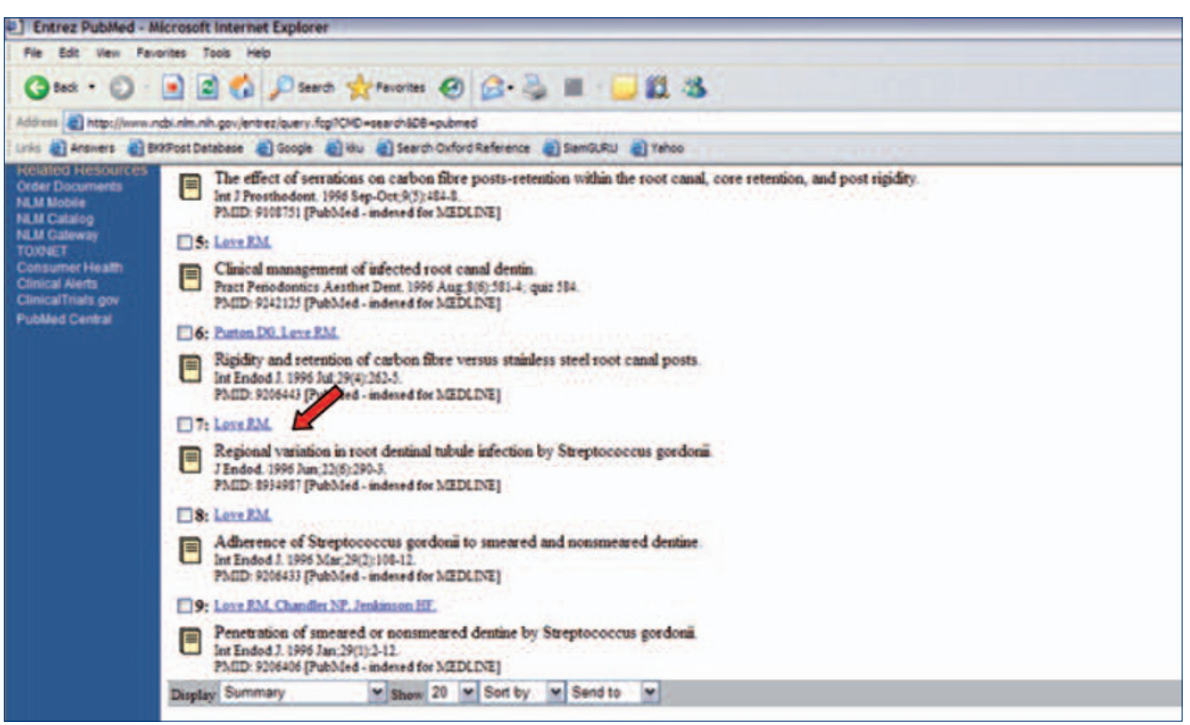

Fig. 4 Article list resulting from the search in Figure 3. See text for explanation 
by numerous laboratory leakage studies, provided instrumentation and root filling are carefully performed. Most bacterial leakage studies can only indicate whether there is bacterial leakage and how soon leakage occurs. Furthermore, one bacterium or 10,000 bacteria differ greatly in terms of disease-causing potential. Another significant point that a laboratory study can never be able to take into account is the host defence mechanism. The human body is extraordinarily complex, and it is impossible to completely and accurately simulate clinical conditions in bench top studies. Although bench top studies have many advantages, clinical studies are more appealing and more relevant, especially to clinicians.

\subsubsection{Clinical studies: what kind?}

Basically, clinical studies test the association between a factor ' $\mathrm{X}$ ' and an event ' $\mathrm{Z} \cdot{ }^{\prime}{ }^{16}$ And if it exists, how strong is that association (Fig. 1)?

\subsubsection{Why is the randomised controlled trial the gold standard?}

This design can minimise confounders, which are 'hidden' variables in a study that affect the variables in question but are not known or acknowledged, and thus (potentially) distort the resulting data. ${ }^{17}$ This design can also maximise control over the environment providing the most convincing causal relationship.

\subsection{Hierarchy of evidence or quality of} evidence (Fig. 1)

The following order is considered: $:^{18}$

1. Systematic review and meta-analysis

2. Randomised controlled trial (RCT)

3. Cohort studies

4. Case control studies

5. Cross-sectional surveys

6. Case series or case reports

7. Expert opinion.

6.5 What is the basis for this hierarchy?

- The systematic review represents the highest quality evidence because it is a summation of the current best (quality checked) individual studies

- The design with randomisation can minimise confounders and selection bias because it leaves only chance to play a role in deciding which subject receives what treatment
- The design with a prospective nature allows researchers to have more control over the environment

- Treatment and disease effects take time to develop, therefore the design with no time element (eg cross-sectional surveys) has limited ability to differentiate between cause and effect

- There is no control group in a case series or case report. It is merely a report of an event, which can be caused by several undetermined factors

- Expert opinion is difficult or virtually impossible to critically appraise.

\subsection{Peer-reviewed journals: why?}

Articles published in peer reviewed journals have been scrutinised by referees, who usually are experts in that particular area. Chances are that seriously flawed studies are less likely to pass through the review process and be published in these journals, but this is not always the case. In view of evidencebased concepts, articles should be critically appraised according to their merits (quality of their study design, materials and methods) rather than in what journal they are published.

'Grey' literature represents articles published in non-peer-reviewed journals eg Dentistry Today, Australasian Dentist. Academically, they are considered low in value. However, in strict adherence to evidence-based concepts, grey literature should be identified and included in the analysis. Such literature can be excluded from analysis only because of merit and merit alone. It should not be excluded because it is not peer-reviewed.

\subsection{How do we know that a journal is peer-reviewed?}

Bowker's Ulrichs website http://www. ulrichsweb.com/UlrichsWeb/ is a website designed for the convenience of librarians regarding purchasing and organising journals. This site contains journal details such as publishers, country of publication, subscription fee, and also whether the journal is refereed or peer-reviewed.

\section{WHERE CAN THE EVIDENCE BE FOUND?}

The significance of locating all available and relevant evidence cannot be overemphasised in the evidence-based concept. The current best available evidence should dictate clinical decision-making and, in fact, is the very foundation of the evidence-based concept. To obtain current best available evidence, all relevant evidence must be located, critically appraised and ranked.

Relying on one or a few studies as the basis for treatment recommendations is not very prudent. A useful analogy is the series of clinical studies comparing the effects of quality of obturation and restoration (apical vs. coronal seal) on healing. In the first such study Ray and Trope ${ }^{19}$ concluded that the quality of the coronal restoration was significantly more important than the quality of endodontic filling to treatment outcome $(\mathrm{P}<0.001$, Chi square test) (odds ratio = 2.6 ; 95\% CI; 1.8 to 3.9). However, a succeeding article ${ }^{20}$ reported the completely opposite result. When five studies investigating the same issue ${ }^{19-23}$ were identified and were statistically combined, a different picture emerged. The highly significant result in Ray and Trope then became non-significant $(\mathrm{P}=0.55)$ with odds ratio of 0.8 (95\% CI; 0.3 to 1.8 ) (meta-analysis data using the random effect method, RevMan Version 4.2.7). Therefore, the quality of the coronal seal is as important as the quality of the apical seal to treatment outcome, and both have an equal impact on prognosis. This emphasises the point that one study is not sufficient, and that all relevant evidence must be identified.

\subsection{Databases}

\subsubsection{The Medline database}

This is a database of biomedical citations and abstracts. Medline covers over 4,800 journals published in the United States and more than 70 other countries primarily from 1966 to the present. Medline includes references to articles indexed with terms from the National Library of Medicine's controlled vocabulary, $\mathrm{MeSH}^{\circledR}$ (Medical Subject Heading). Citations in Medline are from journals selected for inclusion in the database. Essentially, Medline is the most popular and the most comprehensive database for healthcare information, and citation information is electronically accumulated every day. There are several search service providers sharing the same Medline database eg Pubmed, Ovid Medline, SilverPlatter etc. Pubmed is a website providing free services using the Medline database, and is made possible by the National Institute of Health 


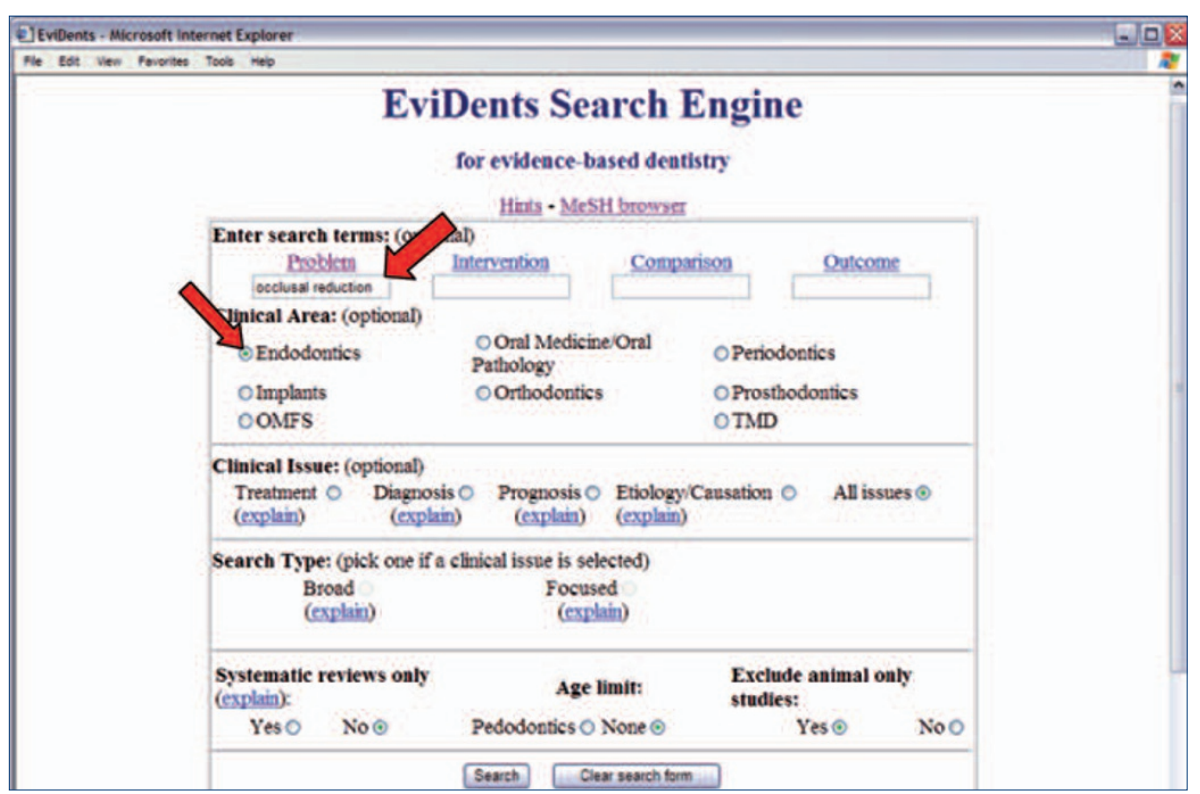

Fig. 5 EviDents search engine website. See text for explanation

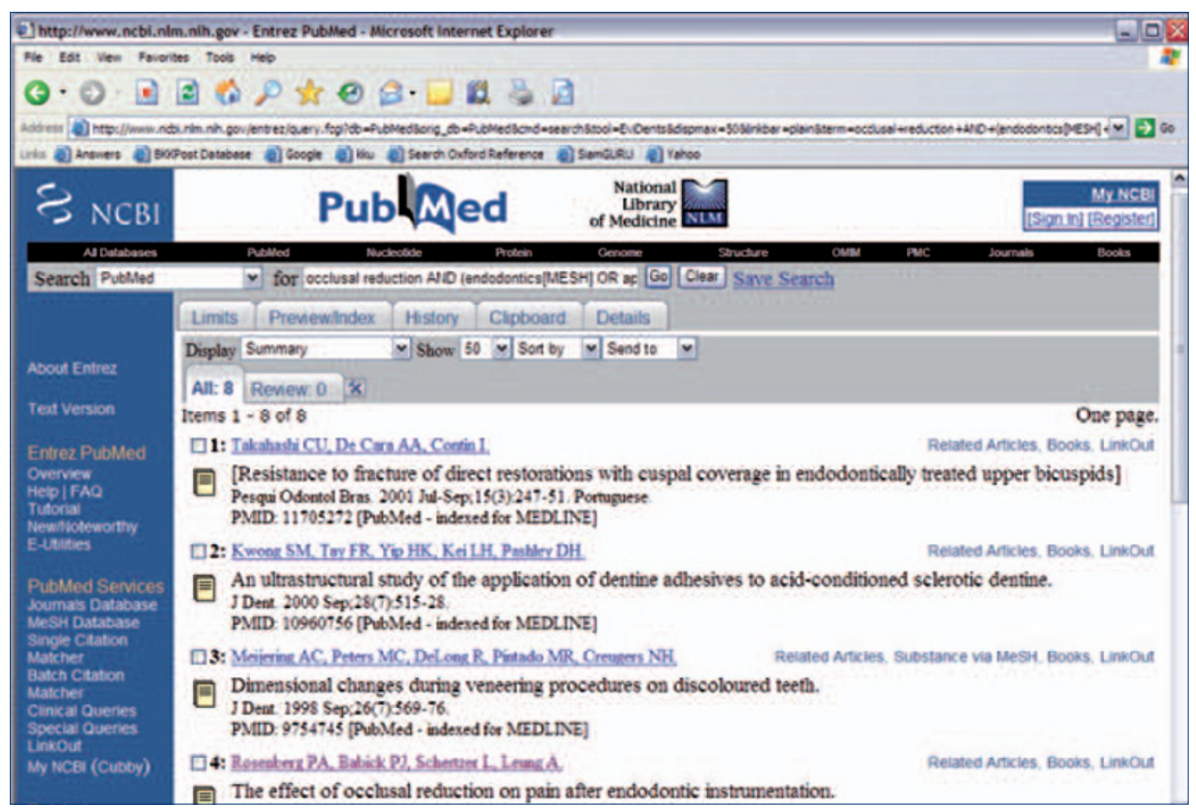

Fig. 6 Result of the search in Figure 5

of the USA. It is the starting point for every search, but for complex search strategies, there are some limitations on Pubmed, and so Ovid Medline may be a better place to run such a search.

\subsubsection{The EviDents search engine http://medinformatics.uthscsa.edu/ EviDents/}

This is a search engine designed specifically for evidence-based dentistry by the University of Texas. It is a complex Medline search strategy made simple by embedding pre-designed search strategies in the EviDents webpage, and the user simply clicks the form. It has benefits from a practical viewpoint, but the benefits are limited, because there are
$34 \%,{ }^{24}$ and it has been shown that using both improves the coverage of the literature. ${ }^{25}$ Embase complements Medline and vice versa. To retrieve important articles that are not indexed in Medline, it is essential to use Embase in searches conducted for a comprehensive review, or to find rare case reports. Embase is more expensive, more time-consuming to use, and perhaps less accessible than Medline. For information about drugs and therapeutics, Embase should be used, and especially when Medline has not retrieved sufficient information or when more comprehensive coverage of the literature is required. From a dental perspective, however, Embase has few advantages over Medline.

\subsubsection{The Science Citation Index database}

A unique feature, which does not exist in other databases, is that this index can find follow-up work done on a key article. This process is called a secondary search. This feature is beneficial when a definitely relevant article (key article) has been located, and subsequent papers can be traced. Chances are that if the later article tried to answer the same questions as the earlier article, the earlier article should be cited by the later one.

\subsubsection{The Cochrane library database}

The Cochrane library provides systematic quality checked summaries of all the evidence on a particular topic. Theoretically, a search should start here because this database contains the most up-to-date and the most methodologically stringent systematic reviews and meta-analyses. Because dentistry was left far behind in the evidence-based movement, currently, this database has little benefit, if any, in the dental field.

Nevertheless, for the sake of completeness and comprehensiveness, all mentioned databases should be searched thoroughly.

\subsubsection{The Embase database}

This is a Medline counterpart, but is a European initiative. It has better coverage of non-English languages and European based journals. Articles in Embase are assigned more index terms than those in Medline, and consequently people using Embase may be less likely to miss an important article but must spend more time browsing through irrelevant material. The overlap of journals in the Medline and Embase databases is about

\subsection{How to locate evidence}

This section summarises the basic technique which has been detailed by a series of articles. . $^{3,26}$

In the Medline database an article is indexed using specific rules by the National Library of Medicine (NLM) of the USA. The indexing information is supplied by publishers and is also generated electronically by NLM. To track 


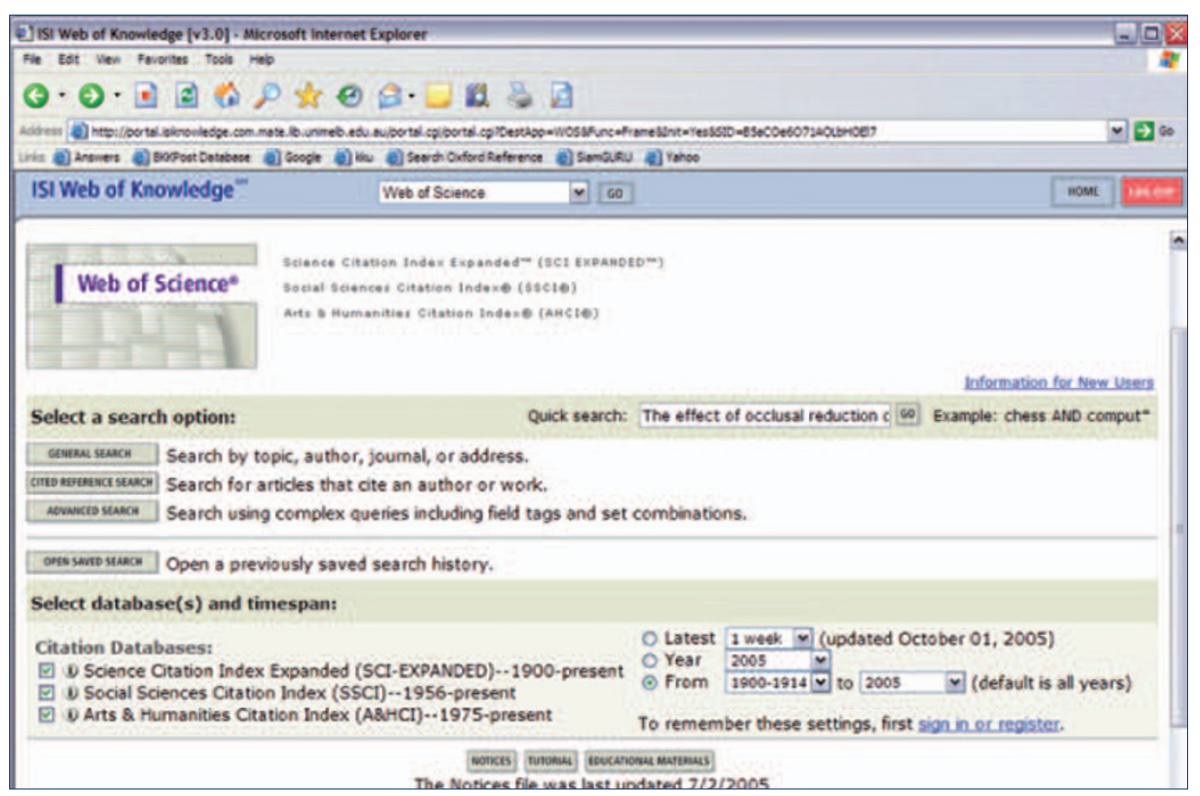

Fig. 7 Use of the Science Citation Index database. See text for explanation

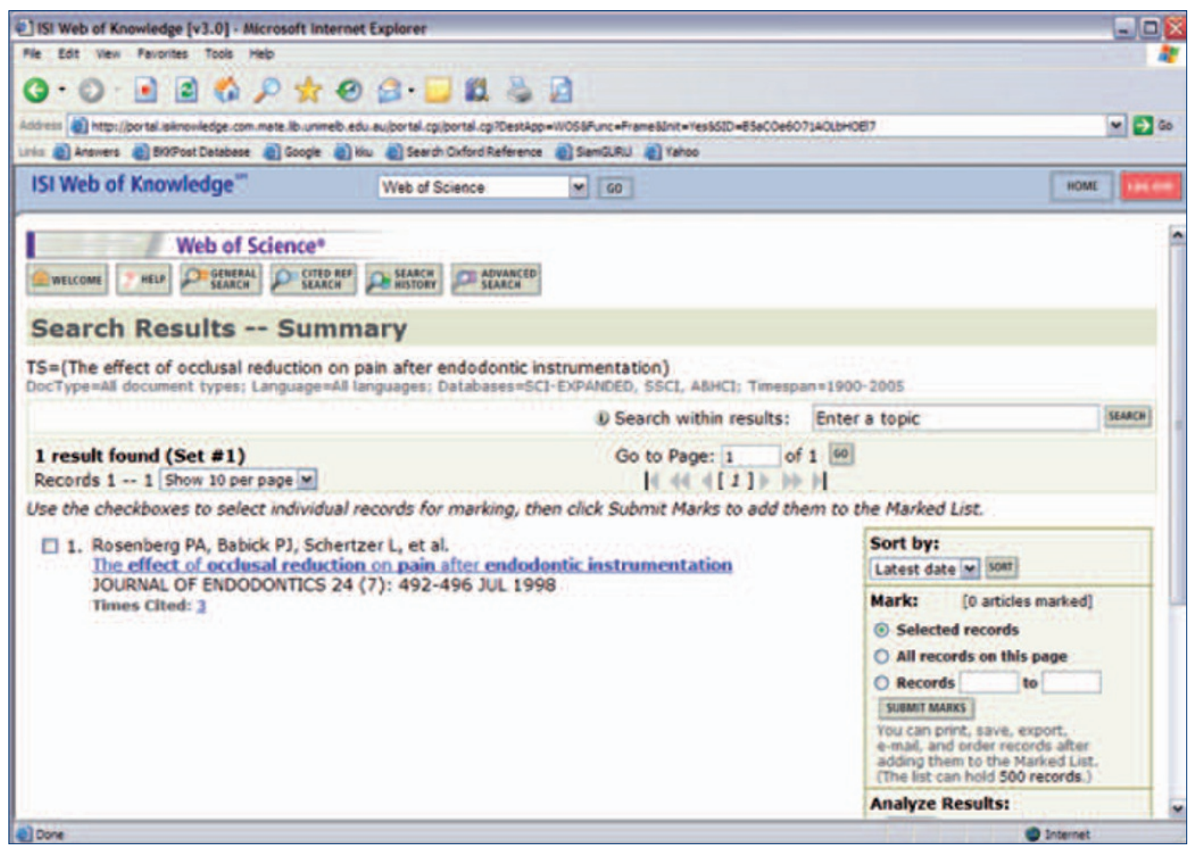

Fig. 8 The use of the Science Citation Index database. See text for explanation

down an article, one needs to guess what words the authors would have used or what words would appear in the citation information. To search comprehensively, all possible words that might be used need to be considered.

Further, search adjuncts, such as Boolean operators (Fig. 2), provide assistance and some control during literature searches. Boolean (named after logician George Boole) is a term used in mathematics, logic and computer science, and represents an expression with two possible values, 'true' and 'false'. The most common Boolean functions are AND, OR and NOT. For example, a 'calcium hydroxide AND bacteria' search strategy will show

\subsubsection{Locating an article already} known to exist

For example, to retrieve the full text article by Love (1996) on how deep bacteria penetrate into dentinal tubules, the following sequence is followed (Fig. 3):

1. Go to the Pubmed website

2. Click 'Limit'

3. Type 'love' in the bar

4. Click the drop down list of 'All Fields' and select 'Author'

5. Enter publication date from 1996 to 1996

6. Click the drop down list of 'Subset' and select 'Dental journals'.

The resulting screen shows nine articles that match the search criteria, and browsing these reveals article number 7 as the required paper (Fig. 4).

\subsubsection{Answering a very specific question}

For example, does routinely reducing the occlusion of teeth undergoing endodontic treatment reduce the chance of interappointment pain? Thus, to find every article that relates occlusal reduction to endodontics, the following sequence is indicated:

1. Go to the EviDents website (Fig. 5)

2. Type 'occlusal reduction' in the 'Problem' bar

3. Click 'Endodontics' in 'Clinical area'

4. Leave the other options in the default settings

5. Click 'Search'

6. The EviDents site will link to the PubMed website showing eight articles (Fig. 6).

After browsing through these articles, the article that is most likely to give an answer would be article number 4 . Namely: The effect of occlusal reduction on pain after endodontic instrumentation. J Endod 1998; 24: 492-496.

The abstract of this paper indicates the study design to be a randomised controlled trial, which provides the most convincing causal relationship between occlusal reduction and pain (or the reduction of pain) after endodontic treatment. Therefore, this could be a key article. To make the search more comprehensive, a secondary search is run on this article in the Science Citation Index database, to determine what articles were cited by this key article and also what articles have cited this key article (Fig. 7). 
1. Copy the title of this key article from the PubMed website

2. Go to the Science Citation Index website

3. Paste the title in the bar at 'Quick search'

4. Click 'GO'

5. This key article will appear, then click on this article (Fig. 8)

6. The next page will show that this key article has 13 references, and this key article was cited by three articles (Fig. 9)

7. Check all 16 articles

8. Two more potentially relevant articles will be detected, which need to be verified by full text only.

- Title: Effect of occlusal relief on endodontic pain

Author(s): Creech JL, Walton RE, Kaltenbach R

Source: Journal of the American Dental Association 109 (1): 64-67 1984

- Title: The effect of occlusal reduction after canal preparation on patient comfort

Author(s): Jostes JL, Holland GR

Source: Journal of Endodontics 10 (1): 34-37 1984

After this comprehensive search, finally, there are three articles to read and from which to obtain information.

\subsubsection{Obtaining general information} quickly about a well defined topic For example, to obtain information about biofilms:

Make use of the $\mathrm{MeSH}^{\oplus}$ terms

1. Go to Pubmed

2. Type biofilm [mh] - any articles which have been indexed in Medical Subject Heading as biofilm will appear. The Medical Subject Heading or $[\mathrm{mh}]$ is the National Library of Medicine's controlled vocabulary used for indexing articles for MEDLINE/PubMed. MeSH terminology provides a consistent way to retrieve information that may use different terminology for the same concepts. The benefit of using MeSH terms is that even if the citation information and abstracts of articles do not contain the word 'biofilm', the articles will still be detected if they are related to biofilm

3. The result is 3,855 articles, which is too many and so the search results

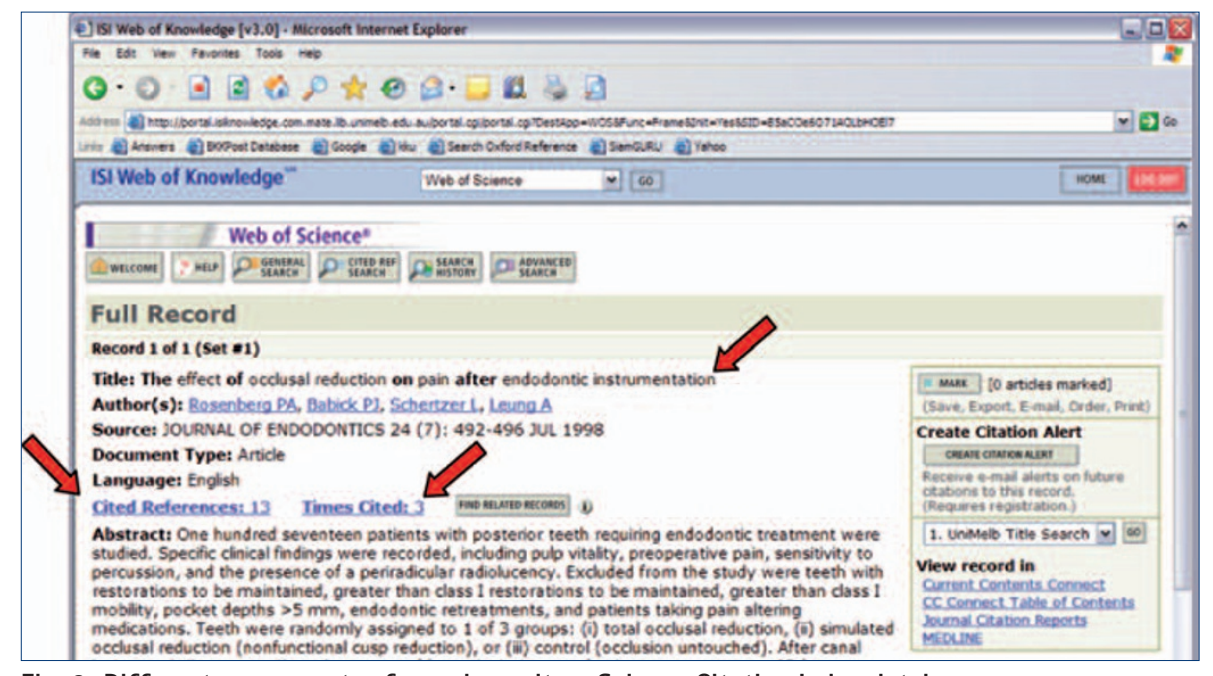

Fig. 9 Different components of search result on Science Citation Index database. See text for explanation

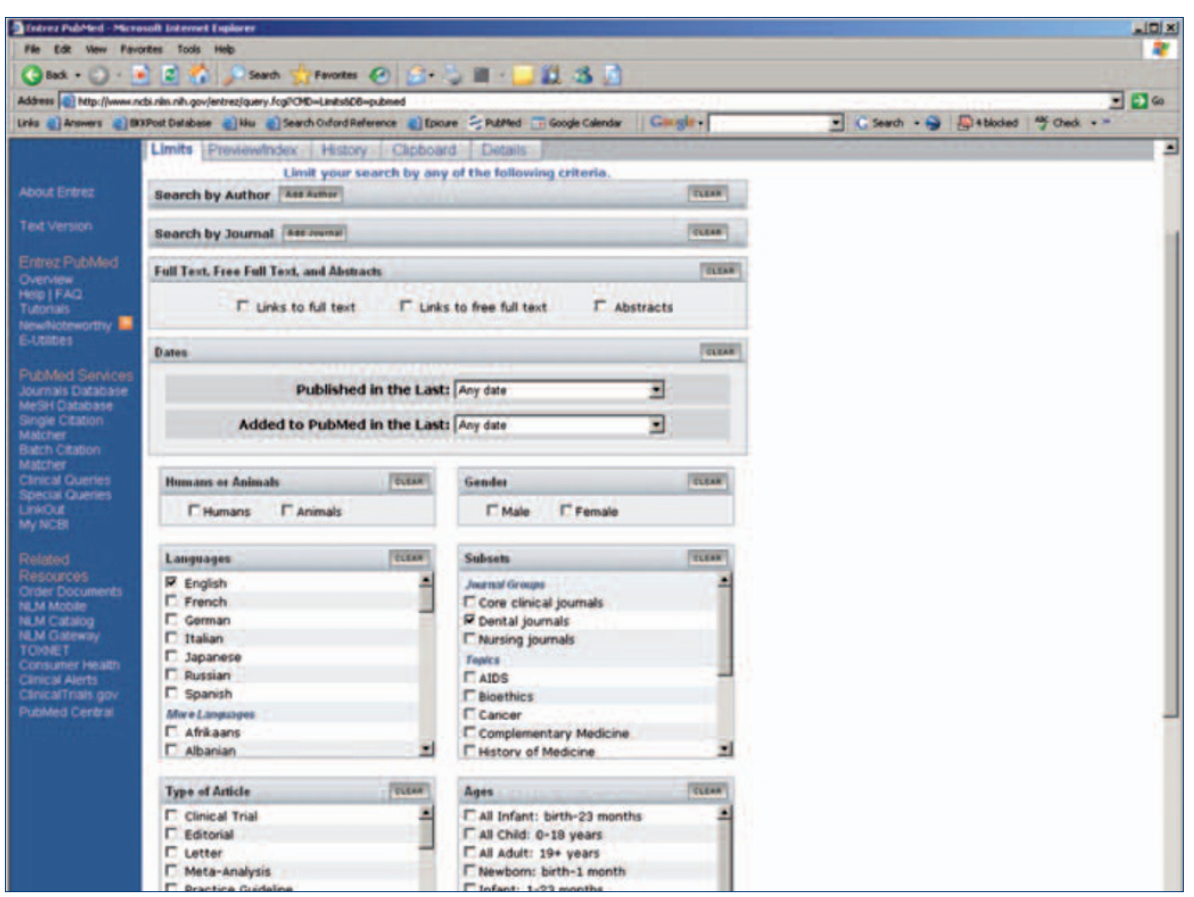

Fig. 10 The use of the 'Limits' feature in the Pubmed website

need to be refined

4. Click 'Limits' (Fig. 10)

5. Click drop down list of 'Type of Article', select 'Review' - any articles that are indexed as review will be detected and shown

6. Click drop down list of 'Languages', select 'English' (or other if appropriate)

7. The result is now 495 articles which is still too many; refine it again

8. Click drop down list of 'Subsets', select 'Dental journals'

9. The result is 88 articles, but this is still too many. If only one article giving general information of biofilm is required, refine the search again.
10. Change biofilm [mh] to biofilm*[ti] - any articles which contain biofilm or biofilms in the title will be detected this time. [ti] is a computer term used here to instruct the search engine to look only in the title.

11. The result is 29 articles, which is now acceptable, and browsing all of them indicates a few potential articles but the most likely key article would be number 13. Namely: Dental biofilms: difficult therapeutic targets. Periodontol 2000 2002; 28: 12-55.

Periodontology 2000 is a journal exclusively for review articles, where authors are invited to submit their 
articles by the editors of each issue. These authors are experts in their fields and their articles are most likely to give a general idea of biofilms.

\subsubsection{What to do when a number of} irrelevant articles are retrieved

In this case a Boolean 'NOT' could be used to exclude irrelevant articles. The initial search strategy in Sathorn et al. ${ }^{4}$ gave hundreds of articles which contained the term single, multiple, appointment and endodontics. Most of them were articles comparing flare-up rates, which, of course, were not relevant. To exclude flare-up studies from the initial results, 'NOT' was added in search strategy by typing 'NOT flare up' in the bar. The result will then be narrowed down.

\section{HOW TO CRITICALLY APPRAISE EVIDENCE}

Once all available evidence has been located, the next step is to ascertain whether the evidence can be trusted and is relevant.

The single most important element of evidence appraisal is probably common sense, which takes time and effort to develop fully. For beginners of evidence appraisal, the following can serve as aids.

\subsection{CASP}

\section{http://www.phru.nhs.uk/casp/casp.htm}

The Critical Appraisal Skill Program (CASP) is a program developed by the National Health Services of the UK. It aims to enable individuals to develop the skills to find and make sense of research evidence, helping them to put knowledge into practice. This program has developed critical appraisal tools for different kind of studies. These tools can be downloaded from http://www.phru. nhs.uk/casp/appraisa.htm.

The tool consists of a series of questions, which are adapted largely from Guyatt et al. ${ }^{27}$ CASP, however, has made the questions succinct, concise and simple for beginners. By trying to answer every question in a critical appraisal tool not only will readers be able to determine the credibility of the paper, but they will understand the paper more thoroughly.

\subsection{CONSORT guidelines}

http://www.consort-statement.org/

A group of scientists, epidemiologists and editors developed the CONSORT
(Consolidated Standards of Reporting Trials) statement, mainly to improve the quality of reporting of RCTs. For a full explanation of CONSORT, please visit the above website or refer to Altman et $a l .{ }^{28}$ Many leading medical journals and major international editorial groups have adopted the CONSORT statement including the Journal of Endodontics and the International Endodontic Journal. The CONSORT statement facilitates critical appraisal and interpretation of clinical study by providing guidance to readers about what should be present in a good quality clinical trial.

\section{CAN THE RESULTS FROM RESEARCH EVIDENCE BE IMMEDIATELY APPLIED? \\ 'Not quite' is probably the answer.}

\subsection{Patient}

First of all, one must ask oneself whether the particular patient would be included in the studies. If this patient will be included, then the conclusion from the evidence can and should be applied to this patient. For example, in Sathorn et $a l .{ }^{4}$ one of the exclusion criteria was retreatment cases. That means that if the patient required endodontic retreatment, then this patient would be excluded from the study, and as a result, conclusions from Sathorn et al. ${ }^{4}$ should not be applied to this patient. Basically, the inclusion and exclusion criteria of the studies should be applied to the particular patient, and if applicable, then the patient may benefit from the conclusions of the evidence.

\subsection{Operator}

Again, the clinician should ask, whether s/he could be one of the operators in the studies. In Sathorn et al., ${ }^{4}$ it is made clear that to apply the conclusions in the paper to one's patients, the operator should be certain to provide the same treatment standard as the operators in the studies. Operator skill will always be an issue because different operators possess different levels of dexterity.

\subsection{Patient's unique values}

Our patients are human and they have preferences. Treatment effectiveness might not be their highest priority. For example, in cancer treatment, a highly effective treatment protocol could deteriorate a patient's quality of life a great deal. A patient might choose quality of life over treatment effectiveness, and we as professionals should respect their autonomy.

\subsection{Patient circumstances}

Identical patient, doctor and environment, but different circumstances could result in different treatment regimens. For example, although MTA apexification lacks clinical evidence supporting its benefits, it could and probably should be the treatment of choice in patients of low compliance especially with time constraints.

\section{SUMMARY AND CONCLUSIONS}

The evidence-based concept is a thought process designed to find the truth, or what is as close to the truth as possible, and makes the full use of it in clinical practice. It makes research evidence and literature more relevant to clinicians. Importantly, it asks more from the clinicians: not only must they master their clinical skills, and manoeuvre their manual dexterities, but also their decision-making has to be logically and scientifically justifiable.

1. Sackett D. Evidence based medicine: how to practice and teach EBM, 2nd ed. Edinburgh: Churchill Livingstone, 2000.

2. Glenny $A M$, Esposito $M$, Coulthard $P$, Worthington $\mathrm{HV}$. The assessment of systematic reviews in dentistry. Eur J Oral Sci 2003; 111: 85-92.

3. Glasziou P. Systematic reviews in health care: a practical guide. Cambridge; New York: Cambridge University Press, 2001.

4. Sathorn C, Parashos P, Messer H H. Effectiveness of single- versus multiple-visit endodontic treatment of teeth with apical periodontitis: a systematic review and meta-analysis. Int Endod J 2005; 38: 347-355.

5. Elderton R J. Clinical studies concerning re-restoration of teeth. Adv Dent Res 1990; 4: 4-9.

6. Goldman M, Pearson A H, Darzenta N. Endodontic success - who's reading the radiograph? Oral Surg Oral Med Oral Pathol 1972; 33: 432-437.

7. Vetter N, Matthews I. Epidemiology and public health. Edinburgh; New York: Churchill Livingstone, 1999.

8. Kakehashi S, Stanley H, Fitzgerald R. The effect of surgical exposures of dental pulps in germ-free and conventional laboratory rats. Oral Surg Oral Med Oral Pathol 1965; 20: 340-349.

9. Möller A J, Fabricius L, Dahlén G, Ohman A E, Heyden G. Influence on periapical tissues of indigenous oral bacteria and necrotic pulp tissue in monkeys. Scand J Dent Res 1981; 89: 475-484.

10. Sundqvist G. Bacteriological studies of necrotic dental pulps. Umea, Sweden: Umea University Odontological Dissertations no. 7, 1976.

11. Sjögren U, Figdor D, Persson S, Sundqvist G. Influence of infection at the time of root filling on the outcome of endodontic treatment of teeth with apical periodontitis. Int Endod J 1997; 30: 297-306.

12. Byström A, Sundqvist $G$. Bacteriologic evaluation of the effect of 0.5 percent sodium hypochlorite in endodontic therapy. Oral Surg Oral Med Oral Pathol 1983; 55: 307-312.

13. Wu M K, Wesselink PR. Endodontic leakage studies reconsidered. Part I. Methodology, application 
and relevance. Int Endod J 1993; 26: 37-43.

14. Torabinejad M, Ung B, Kettering J D. In vitro bacterial penetration of coronally unsealed endodontically treated teeth. J Endod 1990; 16: 566-569.

15. Ricucci D, Grondahl K, Bergenholtz G. Periapical status of root-filled teeth exposed to the oral environment by loss of restoration or caries. Oral Surg Oral Med Oral Pathol Oral Radiol Endod 2000; 90: 354-359.

16. Elwood J M. Critical appraisal of epidemiological studies and clinical trials, 2nd ed. Oxford; New York: Oxford University Press, 1998.

17. McNamee R. Confounding and confounders. Occup Environ Med 2003; 60: 227-234, quiz 164, 234.

18. Greenhalgh T. How to read a paper: the basics of evidence based medicine, 3rd ed. London: BMJ, 2006

19. Ray H A, Trope M. Periapical status of endodontically treated teeth in relation to the technical quality of the root filling and the coronal restoration. Int Endod J 1995; 28: 12-18.

20. Tronstad L, Asbjornsen K, Doving L, Pedersen I, Eriksen $\mathrm{H}$ M. Influence of coronal restorations on the periapical health of endodontically treated teeth. Endod Dent Traumato/ 2000; 16: 218-221.

21. Hommez G M, Coppens C R, De Moor R J. Periapical health related to the quality of coronal restorations and root fillings. Int Endod J 2002; 35: 680-689.

22. Dugas N N, Lawrence H P, Teplitsky P E, Pharoah $M$ J, Friedman S. Periapical health and treatment quality assessment of root-filled teeth in two Canadian populations. Int Endod J 2003; 36: 181-192.

23. Siqueira J F Jr, Rocas I N, Alves F R, Campos L C. Periradicular status related to the quality of coronal restorations and root canal fillings in a Brazilian population. Oral Surg Oral Med Oral Pathol Oral
Radiol Endod 2005; 100: 369-374

24. Smith B J, Darzins P J Ouinn M, Heller R F Modern methods of searching the medical literature. Med J Aust 1992; 157: 603-611.

25. Barillot M J, Sarrut B, Doreau C G. Evaluation of drug interaction document citation in nine on-line bibliographic databases. Ann Pharmacother 1997; 31: 45-49.

26. Greenhalgh T. How to read a paper. The Medline database. Br Med J 1997; 315: 180-183.

27. Guyatt G, Rennie D, Evidence-Based Medicine Working Group, American Medical Association. Users' guides to the medical literature: a manual for evidence-based clinical practice. Chicago, IL: AMA Press, 2002.

28. Altman D G, Schulz K F, Moher D et al. The revised CONSORT statement for reporting randomized trials: explanation and elaboration. Ann Intern Med 2001; 134: 663-694. 